\title{
A four-part boundary value problem in elasticity - indentation by a discontinuously concave punch
}

\author{
J.R. BARBER
}

Department of Mechanical Engineering and Applied Mechanics, University of Michigan, Ann Arbor, MI 48109, USA

\begin{abstract}
A solution is given for the frictionless indentation of an elastic half-space by a flat-ended cylindrical punch with a central circular recess, when the load is large enough to establish a circular region of contact in the recess. The problem is reduced to two simultaneous Fredholm equations using the method of complex potentials due to Green and Collins. Results are presented for the relationship between load, contact radius and penetration for various punch geometries.
\end{abstract}

\section{Introduction}

In the preceeding paper [1], it was shown how the method of complex potential functions due to Green and Collins provides an elegant solution of a wide range of boundary value problems for the half-space. In the present paper we shall use the method to treat an elastic indentation problem which leads to a four-part boundary value problem - the frictionless indentation of an elastic half-space by a rigid, flat-ended cylindrical punch, a central circular region of which is recessed as shown in Fig. 1.

The radius of the punch is $c$ and the central recess has radius $b$ and depth $\epsilon$. The punch is pressed into the half-space to a depth $d$ under the action of an axial force $P$.

For small values of $P$, we anticipate contact only over the annular region $b<r \leq c$ and the resulting problem has been discussed by Collins [2], Shiguya et al. [3] and others. For further discussion of this three-part problem and additional references, see Gladwell [4]. In this paper, we concentrate on the situation when the load $P$ is large enough to establish a circular region of contact whose radius we denote by $a$. Intuitively we anticipate that $a \rightarrow b$ as $P \rightarrow \infty$ and that in the limit $a \rightarrow 0$ we shall recover the solution for the annular punch. 


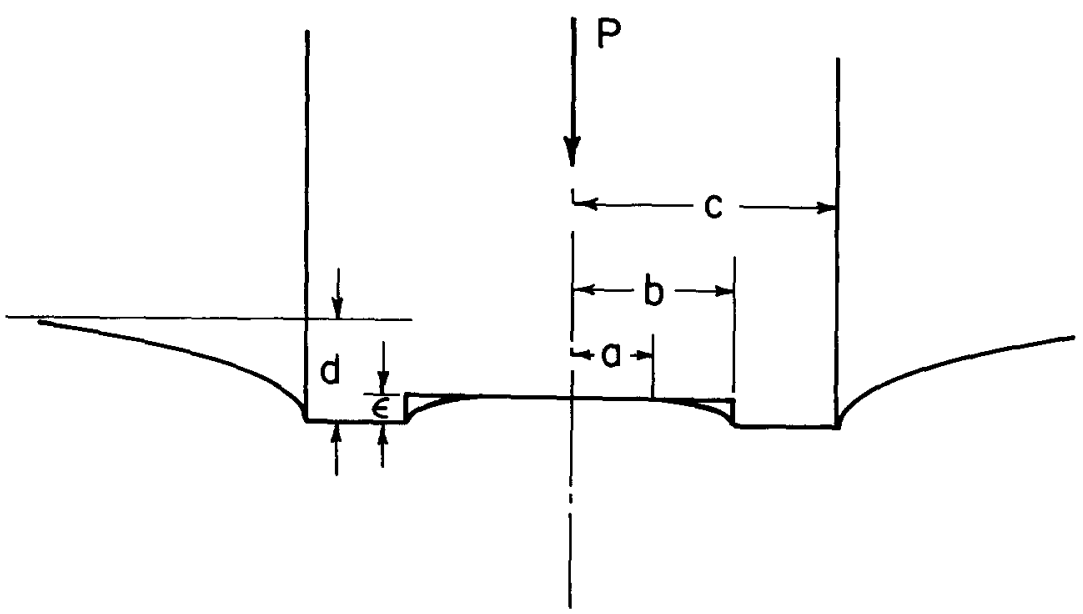

Fig. 1. Indentation of a half-space by a recessed punch

\section{Mathematical formulation}

The boundary conditions of the problem as illustrated in Fig. 1 are

$$
\begin{aligned}
& \begin{aligned}
u_{z} & =(d-\epsilon) ; & & 0 \leq r \leq a \\
& =d ; & & b<r \leq c
\end{aligned} \quad z=0 \\
& \sigma_{z z}=0 ; \quad a<r \leq b \text { and } r>c ; \quad z=0
\end{aligned}
$$

and

$$
\sigma_{r z}=0, \text { all } r ; \quad z=0,
$$

since the contact is assumed to be frictionless.

In view of (3), we use the solution of Eqs. (2)-(4) of [1] with $\psi_{1} \equiv 0$. in which case, the boundary conditions on $\psi_{2}$ become

$$
\begin{aligned}
& \frac{\partial \psi_{2}}{\partial z}=-\frac{\mu(d-\epsilon)}{(1-\nu)} ; \quad 0 \leq r \leq a, z=0, \\
& \frac{\partial^{2} \psi_{2}}{\partial z^{2}}=0 ; \quad a<r \leq b, z=0, \\
& \frac{\partial \psi_{2}}{\partial z}=-\frac{\mu d}{(1-\nu)} ; b<r \leq c, z=0, \\
& \frac{\partial^{2} \psi_{2}}{\partial z^{2}}=0 ; \quad r>c, z=0 .
\end{aligned}
$$




\section{Choice of representation}

As discussed by the present author ([1], Section 8), the general strategy in treating problems with annular regions is to extend the "annular' condition either to $r=0$ or to $r=\infty$ and relax the consequent overprescription by superposition of an additional two-part solution. In the present case, the simplest procedure is to extend (5) to zero and (6) to infinity. However, the extension of (6) to infinity in its present form would lead to ill-conditioned integrals since the representations used force the appropriate derivatives to zero at infinity when the weight functions $g_{i}$ are bounded. We therefore subtract out a classical solution - the Boussinesq indentation solution for a depth of penetration $d$ - before carrying out the extension. We then obtain the solution

$$
\psi_{2}=\phi_{0}+\phi_{1}+\phi_{2}+\phi_{3}+\phi_{4}
$$

where the functions $\phi_{i}$ satisfy the boundary conditions listed in Table 1 .

The solution for $\phi_{0}$ can be obtained easily by standard methods - here we merely record the result

$$
\frac{\partial^{2} \phi_{0}}{\partial z^{2}}=\frac{2 \mu d}{\pi(1-\nu) \sqrt{c^{2}-r^{2}}} ; \quad 0 \leq r \leq c, z=0
$$

which is needed in the subsequent calculations. With this representation it is easily verified that Eqs. (4)-(7) will be satisfied provided

$$
\begin{aligned}
& \frac{\partial \phi_{1}}{\partial z}+\frac{\partial \phi_{3}}{\partial z}+\frac{\partial \phi_{4}}{\partial z}=\frac{\mu \epsilon}{(1-\nu)} ; \quad 0 \leq r \leq a, z=0 \\
& \frac{\partial^{2} \phi_{2}}{\partial z^{2}}+\frac{\partial^{2} \phi_{3}}{\partial z^{2}}+\frac{\partial^{2} \phi_{4}}{\partial z^{2}}=0 ; \quad r>c, z=0 \\
& \frac{\partial^{2} \phi_{0}}{\partial z^{2}}+\frac{\partial^{2} \phi_{2}}{\partial z^{2}}+\frac{\partial^{2} \phi_{3}}{\partial z^{2}}=0 ; \quad 0 \leq r \leq b, z=0,
\end{aligned}
$$

\begin{tabular}{|c|c|c|c|c|c|}
\hline$r=$ & 0 & & & & $\infty$ \\
\hline$\dot{\phi}_{0}$ & & $\frac{\partial \phi_{0}}{\partial z}=-\frac{\mu d}{(1-\nu)}$ & & $\frac{\partial^{2} \phi_{0}}{\partial z^{2}}=0$ & \\
\hline$\dot{\phi}_{1}$ & Eq. (10) & & $\frac{\partial^{2} \phi_{1}}{\partial z^{2}}=0$ & & \\
\hline$\phi_{2}$ & & $\frac{\partial \phi_{2}}{\partial z}=0$ & & Eq. (I1) & \\
\hline$\phi_{3}$ & $\mathrm{Eq} \cdot(12)$ & & & $\frac{\partial \phi_{3}}{\partial z}=0$ & \\
\hline$\phi_{4}$ & $\frac{\partial^{2} \phi_{4}}{\partial z^{2}}=0$ & & Eq. (13) & & \\
\hline
\end{tabular}

Table 1 


$$
\frac{\partial \dot{\phi}_{1}}{\partial z}+\frac{\partial \phi_{4}}{\partial z}=0 ; \quad r>b, z=0
$$

The zero boundary conditions on $\phi_{i}(i=1$ to 4$)$, written explicitly in Table 1, are the same as those satisfied by the corresponding functions in Table 1 of reference [1] and hence they can be satisfied by writing

$$
\left.\begin{array}{l}
\phi_{1}=R \int_{0}^{a} g_{1}(t) F(r, z, t) \mathrm{d} t \\
\phi_{2}=R \int_{c}^{\infty} g_{2}(t) F(r, z, t) \mathrm{d} t \\
\phi_{3}=I_{m} \int_{0}^{b} g_{3}(t) F(r, z, t) \mathrm{d} t \\
\phi_{4}=I_{m} \int_{b}^{\infty} g_{4}(t) F(r, z, t) \mathrm{d} t
\end{array}\right\}
$$

where

$$
F(r, z, t)=\log \left(\left(r^{2}+(z+i t)^{2}\right)^{1 / 2}+z+i t\right) .
$$

Each of the Eqs. (10)-(13) is complementary in range to one of the conditions so satisfied and is used to furnish an Abel equation which can be solved for the corresponding $g_{i}$. Thus, using the results from Table 1 of reference [1] we have

$$
\begin{aligned}
& \int_{0}^{r} \frac{g_{1}(t) \mathrm{d} t}{\sqrt{r^{2}-t^{2}}}=\int_{r}^{b} \frac{g_{3}(t) \mathrm{d} t}{\sqrt{t^{2}-r^{2}}}+\int_{b}^{\infty} \frac{g_{4}(t) \mathrm{d} t}{\sqrt{t^{2}-r^{2}}}+\frac{\mu \epsilon}{(1-\nu)} ; 0 \leq r \leq a \\
& \int_{r}^{\infty} \frac{\operatorname{tg}_{2}(t) \mathrm{d} t}{\sqrt{t^{2}-r^{2}}}=-\int_{0}^{b} \frac{\operatorname{tg}_{3}(t) \mathrm{d} t}{\sqrt{r^{2}-t^{2}}}-\int_{b}^{r} \frac{t g_{4}(t) \mathrm{d} t}{\sqrt{r^{2}-t^{2}}}+C_{1} ; r>c \\
& \int_{0}^{r} \frac{\operatorname{tg}_{3}(t) \mathrm{d} t}{\sqrt{r^{2}-t^{2}}}=-\int_{c}^{\infty} \frac{t_{2}(t) \mathrm{d} t}{\sqrt{t^{2}-r^{2}}}+\frac{\mathrm{d} \sqrt{c^{2}-r^{2}}}{\pi(1-v)}+C_{2} ; 0 \leq r \leq b \\
& \int_{r}^{\infty} \frac{g_{4}(t) \mathrm{d} t}{\sqrt{t^{2}-r^{2}}}=\int_{0}^{a} \frac{g_{1}(t) \mathrm{d} t}{\sqrt{r^{2}-t^{2}}} ; r>b
\end{aligned}
$$

from (10, (11), (12) and (13) respectively. Note that (17) and (18) are obtained after integrating with respect to $r$ and $C_{1}, C_{2}$ are constants of integration. We require continuity of displacement at $r=0$ and hence

$$
C_{2}=\int_{c}^{\infty} g_{2}(t) \mathrm{d} t-\frac{\mathrm{d} c}{\pi(1-\nu)},
$$

from (18). We now write down the solutions of Eqs. (16)-(19) using 
Tables 2 and 3 of reference [1], substituting for $C_{2}$ from (20) to obtain

$$
\begin{aligned}
g_{1}(x)= & -\frac{2}{\pi} \int_{0}^{b} \frac{\operatorname{tg}_{3}(t) \mathrm{d} t}{\left(x^{2}-t^{2}\right)} \\
& +\frac{2}{\pi} \int_{b}^{\infty} \frac{t g_{4}(t) \mathrm{d} t}{\left(t^{2}-x^{2}\right)}-\frac{2 \epsilon \mu}{\pi(1-\nu)} ; \quad 0 \leq x \leq a, \\
g_{2}(x)= & -\frac{2}{\pi} \int_{0}^{b} \frac{\operatorname{tg}_{3}(t) \mathrm{d} t}{\left(x^{2}-t^{2}\right)}+\frac{2}{\pi} \int_{b}^{\infty} \frac{\operatorname{tg}_{4}(t) \mathrm{d} t}{\left(t^{2}-x^{2}\right)} ; \quad x>c, \\
g_{3}(x)= & -\frac{2 x}{\pi} \int_{c}^{\infty} \frac{g_{2}(t) \mathrm{d} t}{\left(t^{2}-x^{2}\right)}-\frac{2 \mathrm{~d} \mu}{\pi^{2}(1-\nu)} \log \left(\frac{c+x}{c-x}\right) ; 0 \leq x \leq b, \\
g_{4}(x)= & \frac{2 x}{\pi} \int_{0}^{a} \frac{g_{1}(t) \mathrm{d} t}{\left(x^{2}-t^{2}\right)} ; \quad x>b .
\end{aligned}
$$

Finally, we substitute (22) and (23) into (20) and (21), obtaining two simultaneous Fredholm equations in $g_{1}, g_{2}$ which are

$$
\begin{aligned}
g_{1}(x)= & \frac{2}{\pi^{2}} \int_{0}^{a}\left[t \log \left(\frac{b+t}{b-t}\right)-x \log \left(\frac{b+x}{b-x}\right)\right] \frac{g_{1}(t) \mathrm{d} t}{\left(t^{2}-x^{2}\right)} \\
& +\frac{2}{\pi^{2}} \int_{c}^{\infty}\left[x \log \left(\frac{b+x}{b-x}\right)-t \log \left(\frac{t+b}{t-b}\right)\right] \frac{g_{2}(t) \mathrm{d} t}{\left(t^{2}-x^{2}\right)} \\
& +\frac{2 \epsilon \mu}{\pi(1-\nu)}-\frac{4 \mathrm{~d} \mu}{\pi^{3}(1-\nu)} \int_{0}^{b} \frac{t \log \left(\frac{c+t}{c-t}\right) \mathrm{d} t}{\left(t^{2}-x^{2}\right)} ; 0 \leq x \leq a \\
g_{2}(x)= & \frac{2}{\pi^{2}} \int_{c}^{\infty}\left[x \log \left(\frac{x+b}{x-b}\right)-t \log \left(\frac{t+b}{t-b}\right)\right] \frac{g_{2}(t) \mathrm{d} t}{\left(t^{2}-x^{2}\right)} \\
& +\frac{2}{\pi^{2}} \int_{0}^{a}\left[x \log \left(\frac{x+b}{x-b}\right)-t \log \left(\frac{b+t}{b-t}\right)\right] \frac{g_{1}(t) \mathrm{d} t}{\left(x^{2}-t^{2}\right)} \\
& +\frac{4 \mathrm{~d} \mu}{\pi^{3}(1-\nu)} \int_{0}^{b} \frac{t \log \left(\frac{c+t}{c-t}\right) \mathrm{d} t}{\left(x^{2}-t^{2}\right)} ; x>c .
\end{aligned}
$$

\section{Load, penetration and contact radius}

Equations (24) and (25) define the solution of the problem in which the penetrations $d, \epsilon$ and the contact radii $a, b, c$ are all prescribed. This 


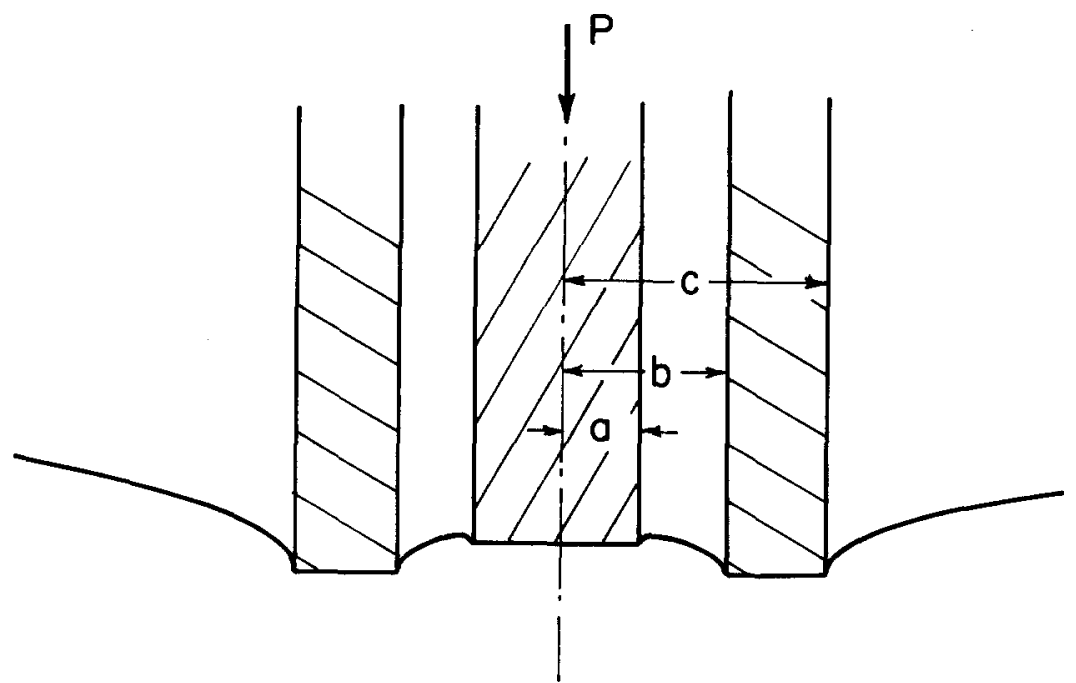

Fig. 2. Indentation of a half-space by an annular punch and a concentric circular punch

would be the case, for example, if a circular punch of radius $a$ and a concentric annular punch were independently pressed into the surface * as illustrated in Fig. 2.

However, for the problem of Fig. 1, the contact radius is not known a priori, but has to be found from the condition that there is no singularity in contact pressure at $r=a$. From Eq. (14) and Table 5 of [1]. we anticipate a sqaure root singularity of strength $g_{1}(a)$ at $r \rightarrow a^{\prime}$ and hence the required condition is

$$
g_{1}(a)=0 \text {. }
$$

To avoid iteration, we interchange the roles of $a$ and $\epsilon$, regarding the former as prescribed and allowing the latter to float, its value being obtained by substituting (26) into (24) with $x=a$, i.e.

$$
\begin{aligned}
\frac{2 \epsilon \mu}{\pi(1-\nu)}= & \frac{2}{\pi^{2}} \int_{0}^{a}\left[t \log \left(\frac{b+t}{b-t}\right)-a \log \left(\frac{b+a}{b-a}\right)\right] \frac{g_{1}(t) \mathrm{d} t}{\left(t^{2}-a^{2}\right)} \\
& -\frac{2}{\pi^{2}} \int_{c}^{\infty}\left[a \log \left(\frac{b+a}{b-a}\right)-t \log \left(\frac{t+b}{t-b}\right)\right] \frac{g_{2}(t) \mathrm{d} t}{\left(t^{2}-a^{2}\right)} \\
& -\frac{4 \mathrm{~d} \mu}{\pi^{3}(1-\nu)} \int_{0}^{b} \frac{t \log \left(\frac{c+t}{c-t}\right) \mathrm{d} t}{\left(t^{2}-a^{2}\right)}
\end{aligned}
$$

* The function $\partial \psi_{2} / \partial z$, defined through Eqs. (8) and (14) can also be used for the electrostatic potential field due to a circular disc and a surrounding annular disc. maintained at different potentials. 
The expression for the load $P$ on the punch is obtained by integrating the expressions for $\partial^{2} \phi_{i} / \partial z^{2}$ over the surface, using Table 4 of [1]. The most natural approach is to perform these integrals only over the loaded region $0 \leq r \leq a$ and $b<r \leq c$, but it proves more efficient to integrate over the whole surface $0 \leq r<\infty$. The same result is obtained, since the only load transmitted across the surface is that exerted by the punch. A further simplification follows from the result mentioned in Section 9 of [1]: that the condition $\partial \phi / \partial z=0$ in an external region (e.g. in $r>b$ ) ensures that the integral of $\partial^{2} \phi / \partial z^{2}$ over the surface is zero. We can use this result to prove that neither $\left(\phi_{1}+\phi_{4}\right)$ nor $\phi_{3}$ make any contribution to the total load because of (13) and Table 1 respectively. Using these results and Table 4 of [1] and Eq. (9), we find that the total load is

$$
P=4 \pi \mu\left[\frac{\mathrm{d} c}{\pi(1-\nu)}+\int_{c}^{\infty} g_{2}(t) \mathrm{d} t\right] .
$$

\section{Numerical solution and results}

For the numerical solution, it is convenient to eliminate the infinite range in Eqs. (24) and (25) by the change of variable $s=c / t$ in (24) and $x=c / y$ in (25). The term in (24) containing $\epsilon$ (now treated as unknown) is eliminated using Eq. (27) and the range of integration is extended as in

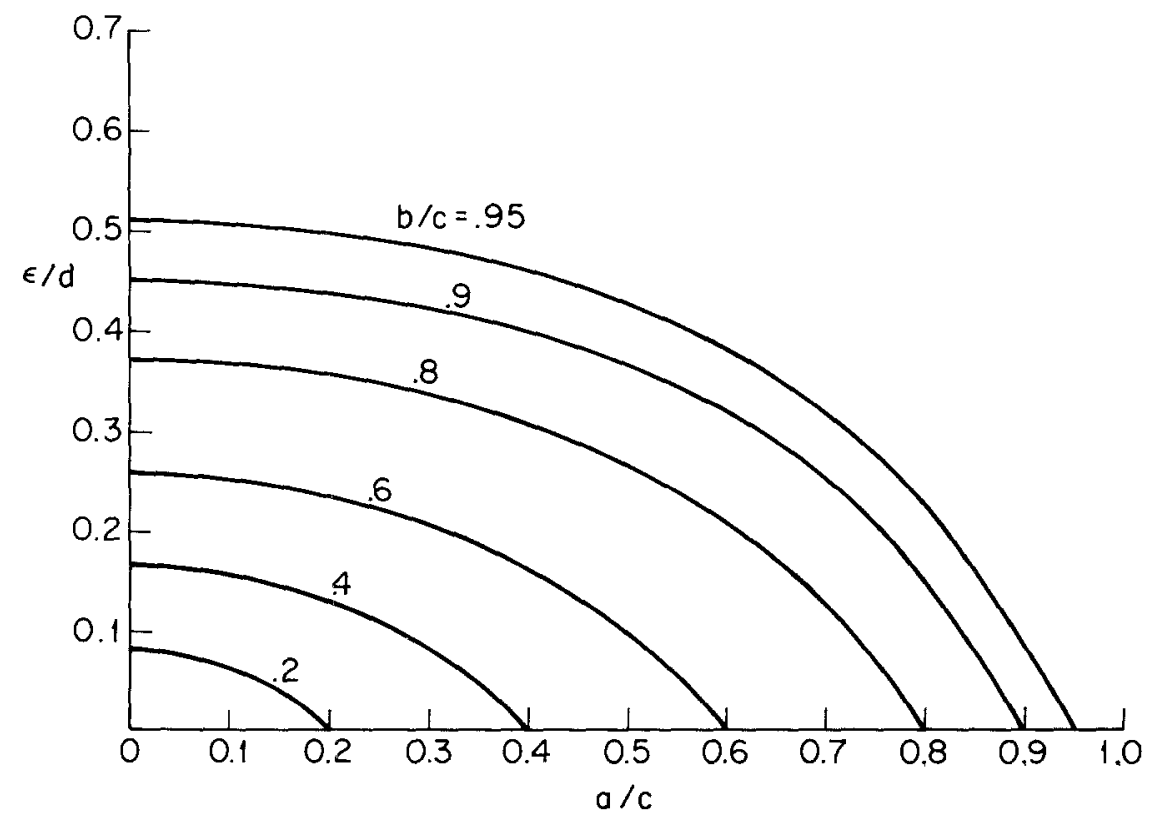

Fig. 3. Contact radius as a function of indentation 


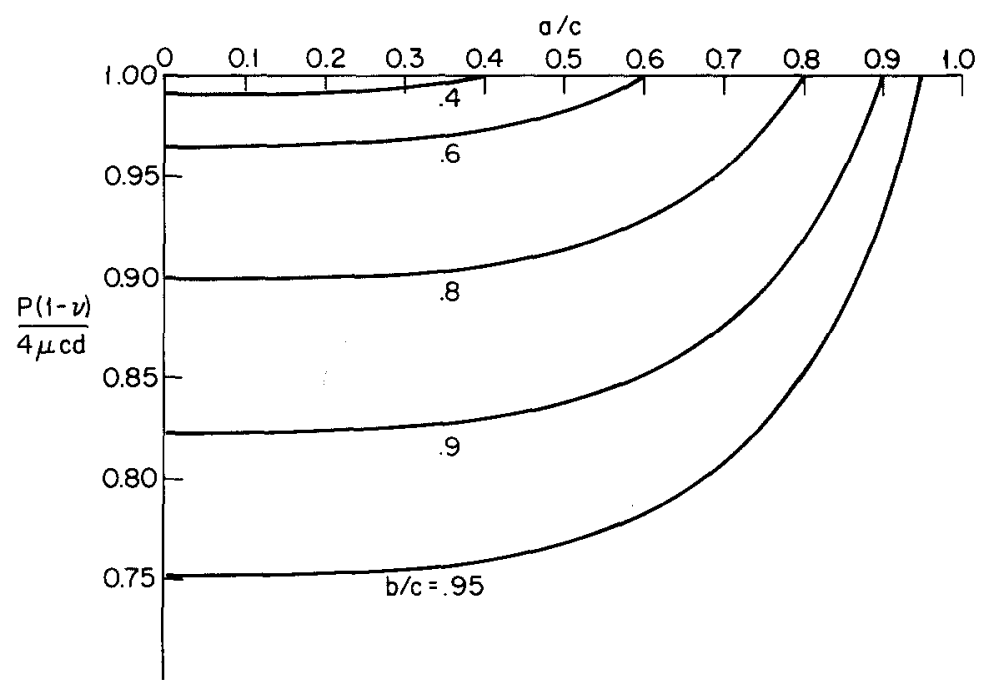

Fig. 4. Ratio between the applied load $P$ and the Boussinesq load $4 \mu c d /(1-\nu)$

Section 7 of [1] resulting in kernels similar to Eq. (47) of [1]. The remaining integrals are then normalized to the range -1 to 1 and the equations are reduced to a system of simultaneous algebraic equations using the Chebyshev quadrature.

Finally, the value of $\epsilon$ is recovered by substitution of the solution for $g_{2}$ into (27) and $P$ is calculated from (28).

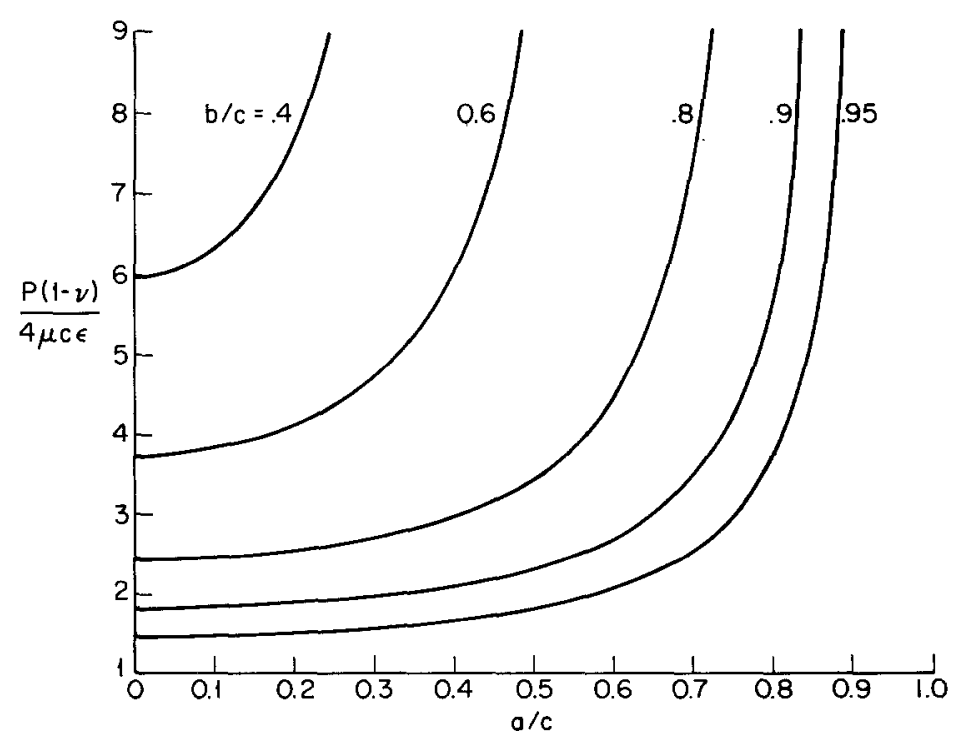

Fig. 5. Load non-dimensionalized with respect to the recess depth $\epsilon$ 
Numerical results for various values of the annular radius ratio $b / c$ are presented in Figs. 3, 4 and 5. Figure 3 shows the relationship between $\epsilon / d$ and $a / c$. Notice that $\epsilon / d$ tends to a limit at $a / c=0$ and hence there is a value of the penetration $d$, below which the geometry of Fig. 1 is impossible. In this range, only the annulus $b<r \leq c$ will make contact with the half-space and the solution is given in [2 and 3]. We should normally regard the geometrical properties of the punch $b, c, \epsilon$ as given, in which case the loading condition is defined through either the penetration $d$ or the applied load $P$. In the former case, the appropriate contact radius can be found from Fig. 3 and the load $P$ from Fig. 4 which represents $P(1-\nu) / 4 \mu c d$ as a function of $a / c$. The non-dimensional load $P(1-\nu) / 4 \mu c d$ is in fact the ratio between $P$ and the Boussinesq load for the same penetration $d$ and we note from Fig. 4 that this ratio is close to unity except when the annulus is thin and the central contact small.

If the load $P$ is specified, $a / c$ can be found from Fig. 5, where $P$ is plotted in the dimensionless group $P(1-\nu) / 4 \mu c \epsilon$. The penetration $d$ is then recovered from Fig. 3.

\section{References}

1. Barber JR (1983) The solution of elasticity problems for the half-space by the method of Green and Collins. App Sci Res 40: 135-157

2. Collins WD (1963) On the solution of some axisymmetric boundary-value problems by means of integral equations: IV. Potential problems for a circular annulus. Proc Edinburgh Math Soc 13:235-246

3. Shibuya T, Koizumi T and Nakahara I (1974) An elastic contact problem for a half-space indented by a flat annular rigid stamp. Int J Engrg Sci 12:759-771

4. Gladwell GML (1980) Contact problems in the classical theory of elasticity. Sijthoff \& Noordhoff, Alphen aan den Rijn, 518-526 